\title{
First results of the NA60 experiment at the CERN SPS
}

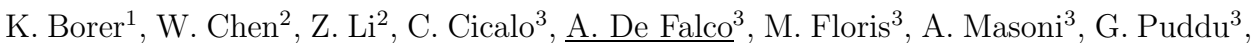
S. Serci ${ }^{3}$, G. Usai $^{3}$, J. Buytaert ${ }^{4}$, M. Keil ${ }^{4}$, C. Lourenço ${ }^{4}$, E. Radermacher ${ }^{4}$, P. Rosinsky ${ }^{4}$, E. Tveiten ${ }^{4}$, J. Castor ${ }^{5}$, A. Devaux ${ }^{5}$, P. Force ${ }^{5}$, N. Guettet ${ }^{5, a}$, F. Manso ${ }^{5}$, K. Banicz ${ }^{6}$, S. Damjanovic ${ }^{6}$, H.J. Specht ${ }^{6}$, A. David ${ }^{7, a}$, J. Lozano ${ }^{7}$, A. Neves ${ }^{7}$, P. Parracho ${ }^{7}$, P. Ramalhete ${ }^{7}$, J. Seixas ${ }^{7}$, R. Shahoyan ${ }^{7}$, P. Sonderegger ${ }^{7}$, R. Veenhof ${ }^{7}$, H. Wöhri ${ }^{4, b}$, B. Cheynis ${ }^{8}$, L. Ducroux ${ }^{8}$, J.-Y. Grossiord ${ }^{8}$, A. Guichard ${ }^{8}$, P. Pillot ${ }^{8}$, R. Tieulent ${ }^{8}$, B. Chaurand ${ }^{9}$, L. Kluberg $9, a$, H. En'yo ${ }^{10}$, J. Heuser ${ }^{10}$, H. Ohnishi ${ }^{10}$, A. Drees ${ }^{11}$, R. Arnaldi ${ }^{12}$, A. Colla ${ }^{12}$, P. Cortese ${ }^{12}$, N. de $\mathrm{Marco}^{12}$, A. Ferretti ${ }^{12}$, C. Oppedisano ${ }^{12}$, E. Scomparin ${ }^{12}$, A. Grigorian ${ }^{13}$, H. Gulkanian ${ }^{13}$, H. Vardanyan ${ }^{13}$

${ }^{1}$ Laboratory for High Energy Physics (LHEP), Bern, Switzerland;

${ }^{2}$ Brookhaven National Laboratory, New York, USA;

${ }^{3}$ Università di Cagliari and INFN (Sezione di Cagliari), Cagliari, Italy;

${ }^{4}$ CERN, Geneva, Switzerland;

${ }^{5}$ LPC, Université Blaise Pascal and CNRS-IN2P3, Clermont-Ferrand, France;

${ }^{6}$ University of Heidelberg, Heidelberg, Germany;

${ }^{7}$ Instituto Superior Técnico, Lisbon, Portugal;

${ }^{8}$ IPN-Lyon, Université Claude Bernard Lyon-I and CNRS-IN2P3, Lyon, France;

${ }^{9}$ LLR, Ecole Polytechnique and CNRS-IN2P3, Palaiseau, France;

${ }^{10}$ RIKEN, Wako, Saitama, Japan;

11 SUNY Stony Brook, New York, USA;

${ }^{12}$ Università di Torino and INFN (Sezione di Torino), Turin, Italy;

${ }^{13}$ YerPhI, Yerevan Physics Institute, Yerevan, Armenia;

${ }^{a}$ Also at CERN;

${ }^{b}$ Now at IST;

The NA60 experiment at the CERN SPS studies dimuon production in proton and heavy ion collisions. Its silicon pixel vertex telescope allows tracking of muon pairs in the vertex region, complementing the information from the muon spectrometer, and improving the mass resolution and signal-to-noise ratio. First results from the In-In data taking are shown for the light resonances and the $\mathrm{J} / \psi$ meson production.

Dileptons are generally considered as interesting probes in the study of heavy ion collisions. Key features as the modification of the $\rho$ meson mass shape due to chiral symmetry restoration, the production of thermal dileptons and the melting of charmonia states, detected through their decays into muon pairs, are accessible through this channel.

The CERES experiment observed that the $e^{+} e^{-}$mass spectrum in the range between 200 and $600 \mathrm{MeV}$ produced in $\mathrm{S}-\mathrm{Au}$ and $\mathrm{Pb}-\mathrm{Au}$ collisions exceeds the expected conventional sources, namely the light meson decays into dielectrons [1]. This observation 
may be associated to the approach to chiral symmetry restoration in the hot and dense medium created in heavy-ion collisions. However, this result suffers from poor statistics and signal-to-background ratio.

In the mass region between 1.5 and $2.5 \mathrm{GeV} / \mathrm{c}^{2}$ the NA38 and NA50 experiments observed an excess in the data collected in $\mathrm{S}-\mathrm{U}$ and $\mathrm{Pb}-\mathrm{Pb}$ collisions when compared to the superposition of Drell-Yan and open charm processes, which describe the mass spectra in p-A collisions [2]. The origin of the excess, which may be due to thermal dimuon production or to the enhancement of charm production, is still not clarified since NA38/50 cannot discriminate between prompt dimuons and muon pairs originating from the $\mathrm{D}$ meson semileptonic decays, characterized by displaced vertices. A spatial resolution of $\sim 50 \mu \mathrm{m}$ in the transverse direction is needed to separate these two contributions.

The NA50 collaboration observed an anomalous suppression of the $\mathrm{J} / \psi$ meson as a function of centrality, when compared to the extrapolation of the p-A data [3]. One of the possible interpretations consists in the melting of the $\chi_{c}$ meson in a deconfined medium, leading to the disappearance of a significant fraction $(\sim 30 \%)$ of $\mathrm{J} / \psi$ coming from the $\chi_{c}$ decays. However, some points are still unclear. Is the $\mathrm{J} / \psi$ suppression due to the dissociation in a quark-gluon plasma or in a deconfined (but not thermalized) medium? What is the variable driving the $\mathrm{J} / \psi$ suppression? Is it the energy density, or the mean path $\mathrm{L}$ of the charmonium in the nuclear matter traversed, or the number of participant nucleons?

The NA60 experiment is designed to clarify some of these open questions. Its apparatus, displayed in Fig. 1 and 2, complements the zero-degree calorimeter (ZDC) and muon spectrometer previously used in the NA50 experiment with a new vertex region using two state-of-the-art silicon detectors: a silicon pixel telescope and a silicon beam tracker.

The ZDC [4], a quartz-fiber "spaghetti" calorimeter, measures the energy carried by spectator beam nucleons, and is used to determine the centrality of the collision.

The muon spectrometer is composed of an air-core toroidal magnet, eight multi-wire proportional chambers for muon tracking and four scintillator hodoscopes that provide the trigger signal. A hadron absorber, placed between the target region and the spectrometer, ensures that only muons can trigger the experiment. On the other hand, because of multiple scattering and energy loss, it limits the dimuon mass resolution and makes an accurate vertex determination impossible.

The silicon pixel telescope is used to track charged particles in the vertex region. It consists of several tracking planes placed immediately after the target, inside a $2.5 \mathrm{~T}$ dipole magnet. For the Indium run the system provided 12 tracking points. The planes are made from assemblies of a silicon sensor chip bump-bonded to a radiation tolerant readout chip, developed for the ALICE and $\mathrm{LHCb}$ experiments. They have a $256 \times 32$ matrix of $50 \times 425 \mu \mathrm{m}^{2}$ cells covering a sensitive area of $12.8 \times 13.6 \mathrm{~mm}^{2}$ [5]. Tracking in the vertex region significantly improves the $\mu \mu$ mass resolution and the signal-to noiseratio, the former by matching muon tracks before and after the absorber and the latter by rejecting muons from $\pi$ and $K$ decays, the main source of background.

The beam tracker [6], placed upstream of the target, is a cryogenic silicon microstrip detector, used to track the incoming beam particles before they hit the target. It is composed of four sensors arranged in two tracking stations, which operate at $130 \mathrm{~K}$ in order to increase the radiation hardness. Its signals are read by a multi-hit time recorder 


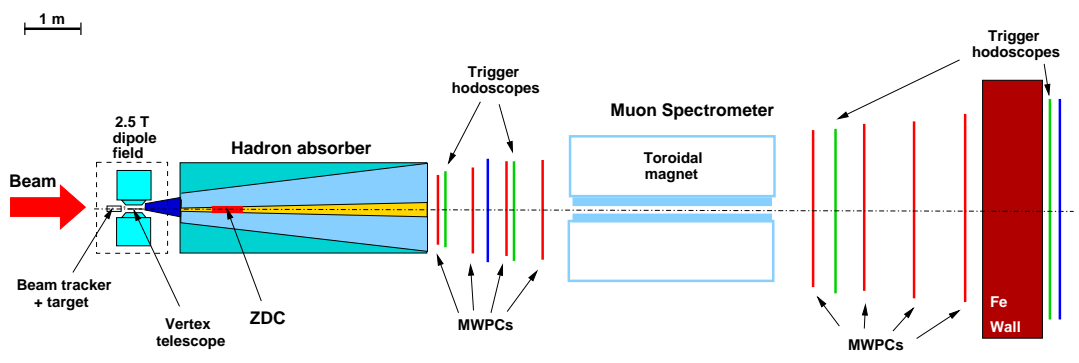

Figure 1. Layout of the NA60 apparatus.

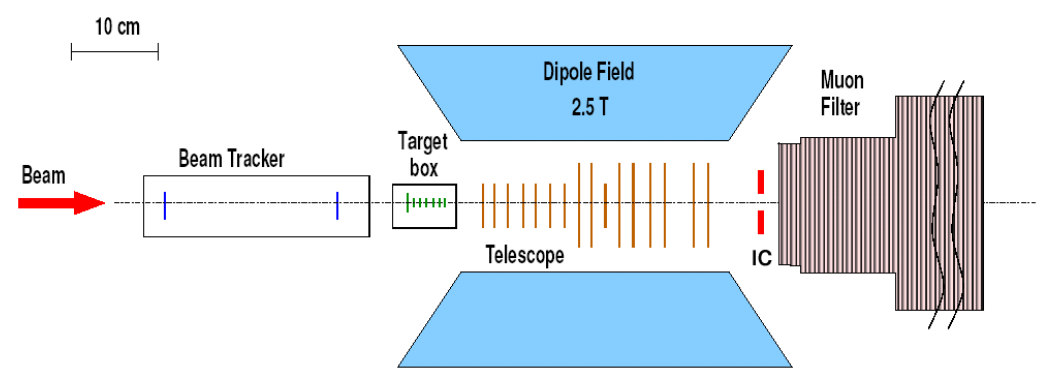

Figure 2. Target region in detail.

unit that measures the time difference between each hit in the strips and the trigger with an accuracy of 1.7 ns.

An interaction counter, composed of two plastic scintillators, is placed just before the hadron absorber. It detects the collisions occurred in the target. The target is placed in vacuum in order to minimize the probability of interactions with air.

During the 5 -week long Indium run in $2003,4 \cdot 10^{12}$ ions, with an energy of $158 \mathrm{AGeV}$ were delivered, and a sample of $2.3 \cdot 10^{8}$ dimuon events was written on tape.

In Fig. 3 the $z$ coordinate of the interaction point is shown. The seven Indium targets between the two target box windows can be clearly distinguished. The spatial resolution in this coordinate is around $200 \mu \mathrm{m}$, while in the transverse plane it is $\sim 20 \mu \mathrm{m}$.

The mass resolution at $\sim 1 \mathrm{GeV}$ is around $20-25 \mathrm{MeV}$, as shown in Fig. 4, where the invariant dimuon mass in the region of the $\omega$ and $\phi$ resonances is plotted for a sample consisting of around $1 \%$ of the total statistics. The background due to uncorrelated decays of pions and kaons into muons was estimated through a mixed-event technique. The acceptance is sizable still at very low transverse masses, thus making possible an analysis of the low mass dilepton production as a function of transverse momentum. The high statistics minimum bias sample permits a study on a wide centrality range. In 


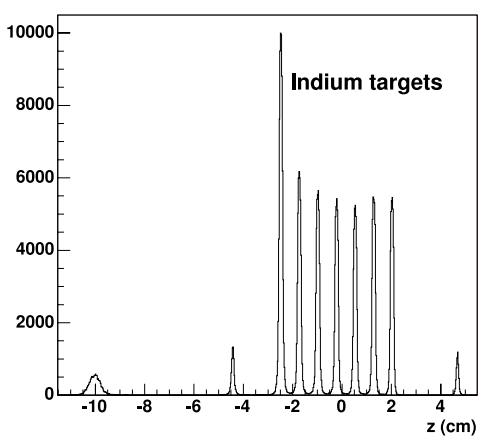

Figure 3. Distribution of the interaction point $z$ coordinate.

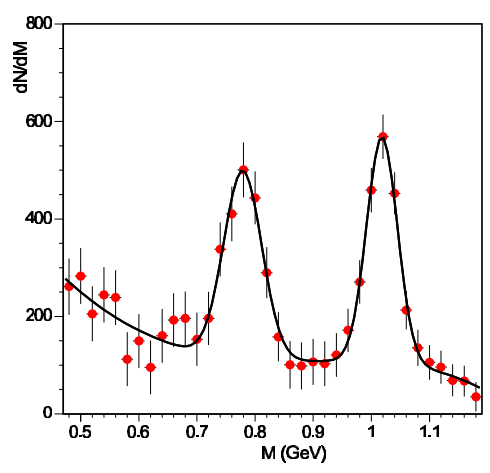

Figure 4. Invariant mass spectrum in the region of the $\omega$ and $\phi$ resonances.

Fig. 5 the mass spectrum at low masses is shown for three multiplicity intervals. The contribution from fake matches, i.e. muon tracks in the spectrometer incorrectly matched with the charged tracks in the vertex telescope, is not yet subtracted. It can be noticed that the $\omega$ peak is visible even at most central collisions.

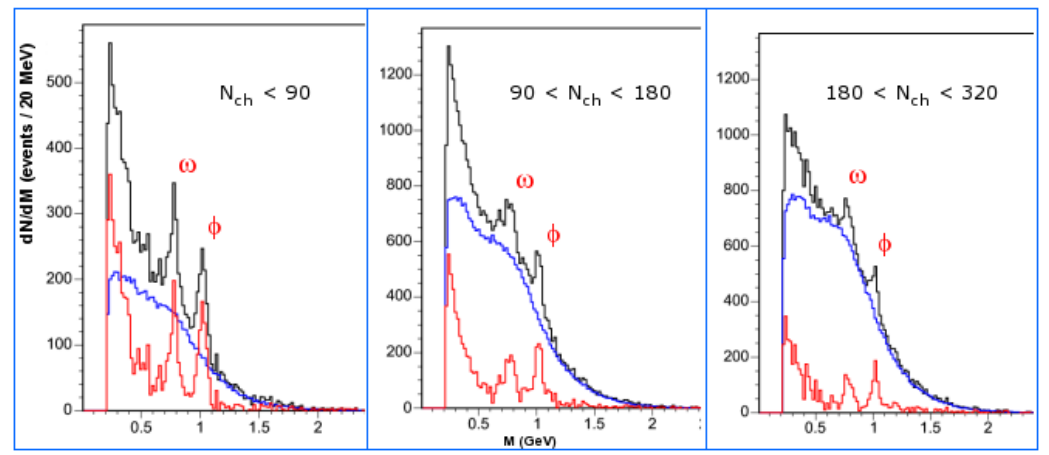

Figure 5. Invariant mass spectrum for three multiplicity intervals. The mass spectrum for the opposite sign muon pairs, the combinatorial background, and the signal obtained after background subtraction are plotted.

A preliminary analysis of the $\mathrm{J} / \psi$ production has been performed. The sample amounts to about one half of the total statistics collected. Events with two (or more) ions having a time difference lower than the ZDC acquisition gate were rejected using the beam 
tracker timing information. Non-interacting beam ions were rejected using the interaction counter. Events with $E_{Z D C}<15 \mathrm{TeV}$ were selected. At this stage of the analysis, the muon pairs were reconstructed using only the information from the muon spectrometer. Events in which the muon tracks did not point to the target were rejected. The analysis was limited to dimuons in the phase space window $0<y_{C M}<1$ and $\left|\cos \theta_{C S}\right|<0.5$, where $\theta_{C S}$ is the muon polar angle, calculated with respect to the beam direction, in the dimuon rest frame.

The dimuon mass spectrum (Fig. 6) was described as a superposition of the $\mathrm{J} / \psi$ and $\psi^{\prime}$ resonances, the open charm and Drell-Yan processes contributions, and the combinatorial background, determined from the measured like-sign muon pairs. The physical processes were simulated using Pythia with MRS-A (low $Q^{2}$ ) parton distribution functions. The detector response was simulated using GEANT 3.21. The fit was performed with a multistep technique: in the first step, the fit was executed for $M>4.2 \mathrm{GeV}$, in order to determine the normalization for the Drell-Yan process. In the second step, the mass spectrum in the range $2.2<M<2.5 \mathrm{GeV}$ was fitted, keeping the Drell-Yan normalization fixed, and determining the open charm normalization. Finally, a fit in the range $2.9<$ $M<4.2 \mathrm{GeV}$ was performed to get the $\mathrm{J} / \psi$ and $\psi^{\prime}$ yields. The ratio between the cross sections of the $\mathrm{J} / \psi$ and the Drell-Yan process (calculated between 2.9 and $4.5 \mathrm{GeV}$, for comparison with previous results) was determined: $\sigma_{J / \psi} / \sigma_{D Y}=19.5 \pm 1.6$. This result is shown in Fig. 7, together with the previous NA38, NA51 and NA50 measurements, reported as a function of $\mathrm{L}$. The uncertainty includes only the statistical error. The evaluation of the systematic effects is ongoing, together with several improvements in the analysis procedure.

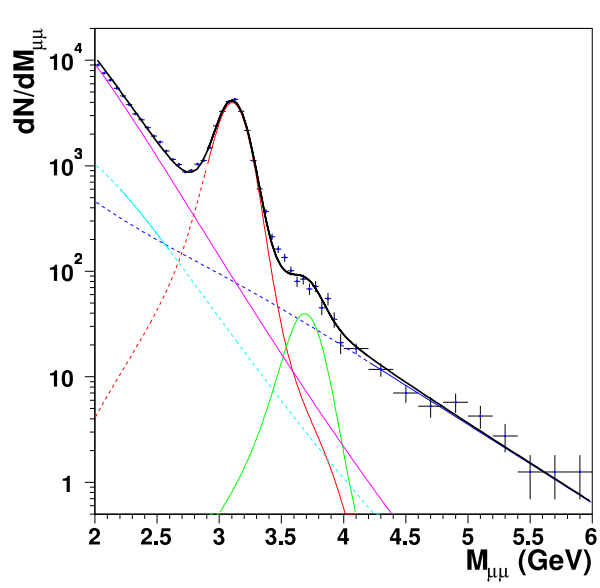

Figure 6. Dimuon mass distribution in the high mass region.

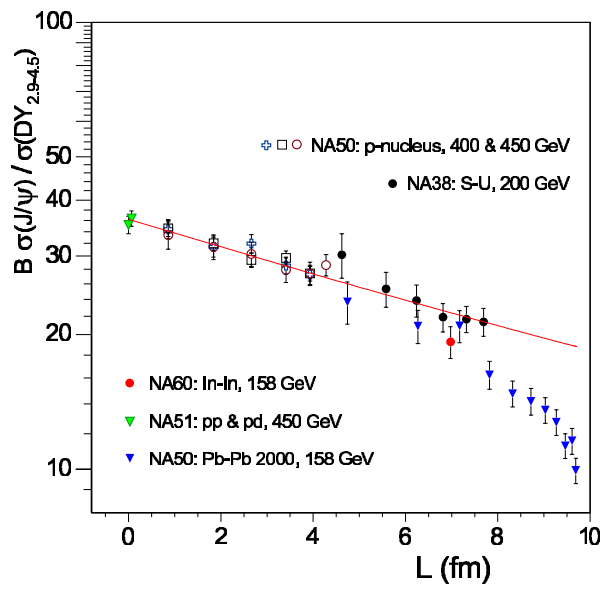

Figure 7. $\mathrm{J} / \psi$ suppression pattern as a function of the mean path of the charmonium in the nuclear matter transversed. 
The measurements in proton-nucleus collisions provide the baseline for the understanding of heavy ion data, and must be exploited with high accuracy. A high statistics run, currently being performed, will provide the reference for the low mass region with unprecedented precision, and allow the direct measurement of open charm and Drell-Yan processes. Also, the $\chi_{c} \rightarrow J / \psi+\gamma$ decay will be studied for different nuclear targets.

\section{REFERENCES}

1. G. Agakichiev et al. (CERES Coll.), Phys. Lett. B422 (1998) 405; Phys. Rev. Lett. 75 (1995) 1272.

2. M.C. Abreu et al. (NA50 Coll.), Eur. Phys. J. C14 (2000) 443.

3. M.C. Abreu et al. (NA50 Coll.), Phys. Lett. B410 (1997) 337.

4. R. Arnaldi et al., Nucl. Instr. Meth. in Phys. Res. A 411 (1998) 1.

5. M. Keil et al., "Operation and Performance of the NA60 Silicon Pixel Telescope", CERN-PH-EP-2004-021, submitted for publication in Nucl. Instrum. Meth. A.

6. P. Rosinsky et al. (NA60 Collaboration), Nucl. Instr. Meth. A 511 (2003) 200. 